\title{
La dialisi domiciliare peritoneale
}

\author{
Valentina Paris
}

Presidente A.N.E.D., A.N.E.D. Associazione Nazionale Emodializzati - Dialisi e Trapianto - Onlus, Milano

\begin{abstract}
HOME PERITONEAL DIALYSIS
Abstract. Peritoneal dialysis is the right treatment option for patients who can clinically handle the daily needs of this therapy, who are well informed and decide to dialize at home.

So far I experienced that patients who agree to do peritoneal dialysis and understand the numerous benefits associated, are mostly young patients waiting for a transplant in the near future (within two years), old people who still have a partner and women (usually housewives), who prefer staying at home and take care of their families.
\end{abstract}

Conflict of interest: None.

Financial support: None.

Accettato: 20 Gennaio 2014

La malattia renale in fase terminale è una condizione anomala: in generale, il malato deve fare i conti con importanti cambiamenti che richiedono tempo, pazienza, sacrifici e rinunce e, nello specifico, deve seguire una nuova dieta, assumere costantemente farmaci e vedere il proprio corpo cambiare. Tuttavia, questi cambiamenti si possono affrontare adeguatamente attraverso una buona informazione iniziale, che permetta al malato di scegliere il trattamento renale sostitutivo e la terapia più consoni al proprio stile di vita.

Di fatto, già nel 1979, la fondatrice di ANED (Associazione Nazionale Emodializzati), Franca Pellini, stilando la Carta dei Diritti e dei Doveri del Malato, scriveva che ogni paziente ha il diritto all'informazione e alla conoscenza necessarie per avere consapevolezza del proprio stato e per poter operare coscienziosamente delle scelte e il dovere di ricercare tutte le restanti informazioni per una gestione responsabile della propria condizione morbosa, perché una buona informazione gli permetterà di adattare la malattia alla vita e non la vita alla malattia.

Questo presuppone l'assistenza di un team (sanitario) multidisciplinare dedicato e formato in ogni unità operativa di nefrologia e dialisi, che possa garantire un'informazione strutturata e costante ai malati nefropatici in procinto di iniziare il trattamento sostitutivo della funzione renale, al fine di individuare il più adatto per ciascun paziente, dal punto di vista non solo clinico, ma anche sociale. Poter fare una dialisi che meglio risponda alle esigenze della persona malata fa ragionevolmente supporre che il recupero delle sue dimensioni fisica, psicologica e sociale sarà veloce e migliore.

Tra le diverse dialisi a cui ci si può sottoporre, la peritoneale è quella che meno colpisce la vita del paziente, perché si effettua indipendentemente in casa. Ricordo che, durante la mia esperienza di infermiera professionale, compresi a fondo cosa significasse la dialisi peritoneale domiciliare la prima volta che sono stata a casa di una paziente, Raffaella.

Era una giovane mamma malata, in dialisi da poco, e, quando sono arrivata, lei era in camera da letto, indossava una tuta e stava giocando con sua figlia sul letto. Dal materiale sparso vicino a loro si capiva che stavano giocando già da un po': Barbie, libri di fiabe, costruzioni Lego... Solo in un secondo momento mi sono accorta che Raffaella stava facendo dialisi! Era, infatti, "attaccata" alla macchina della peritoneale automatizzata, sistemata accanto al letto, quasi nascosta, e non si vedeva immediatamente.

Raffaella faceva parte di quel gruppo di pazienti che avevano capito $\mathrm{i}$ grandi vantaggi e le potenzialità della dialisi peritoneale e, con lei, molte altre mamme casalinghe che potevano, così, continuare a prendersi cura della famiglia, giovani ragazzi in attesa di trapianto che, grazie alla peritoneale notturna, potevano continuare ad andare a scuola regolarmente, nascondendo facilmente quel tanto odiato tubicino sull'addome, e, ancora, anziani in discrete condizioni cliniche che, con l'aiuto del partner, evitavano di andare in ospedale tre volte o più a settimana, come la Signora Maria.

Appena arrivava in ambulatorio per il controllo mensile della funzione renale, doveva stendersi sul letto un paio di ore, prima di riuscire a riprendere fiato e le forze necessarie per fare il prelievo e la visita. Abitava in un paesino dell'alta Valle Imagna, a $40 \mathrm{~km}$ dall'ospedale, e ogni volta il tortuoso viaggio da casa all'ospedale le provocava nausea, vomito e cefalea. Il solo venire a fare i controlli una volta al mese per lei era un calvario, quindi il suo medico non si sorprese del fatto che, una volta informata che avrebbe dovuto iniziare la dialisi, rifiutò la possibilità di quella terapia, che l'avrebbe costretta a recarsi in ospedale tre volte a settimana. Di fronte alla sua determinazione di rinunciare alla dialisi, lo staff le propose, in- 
fine, la peritoneale, nonostante alcuni dubbi su come avrebbe potuto occuparsene. Maria, infatti, risultava essere un'anziana signora fragile, agli occhi delle infermiere che la accoglievano in ospedale per i controlli. Quando, però, iniziarono il training alla CAPD, in casa, conobbero una Maria differente. La paziente non era la paziente che lo staff pensava che fosse, bensì una donna attiva, che si occupava della casa e della famiglia, che cucinava, che curava i nipoti dopo la scuola e che lavorava a maglia... Infatti, imparò velocemente e bene a fare la dialisi peritoneale in casa e, durante i 7 anni di terapia, non ebbe mai un'infezione.

Questa esperienza mi ha insegnato che i malati con cui si interagisce in ospedale non si conoscono mai nella loro interezza. Se ne conosce il quadro clinico, ma non si conoscono il loro ruolo in famiglia e nella società, le loro competenze o le loro aspettative, pertanto diventa indispensabile che l'operatore sanitario abbia il tempo per conoscere le caratteristiche dei malati in pre-dialisi e che il malato partecipi attivamente alla scelta della terapia sostitutiva.

\section{Riassunto}

La dialisi domiciliare peritoneale è un'ottima terapia per pazienti che clinicamente possono far fronte ai bisogni quotidiani di questo tipo di terapia, che sono ben informati e che decidono di dializzarsi in casa. Finora ho sperimentato che i pazienti che accettano di fare la dialisi peritoneale $\mathrm{e}$ che ne comprendono i numerosi benefici associati sono soprattutto giovani pazienti in attesa di un trapianto nel futuro immediato (entro due anni), anziani che hanno ancora un partner e donne (solitamente casalinghe) che preferiscono stare a casa e occuparsi della propria famiglia.

Parole chiave: Dialisi domiciliare, Educazione pre-dialisi, Compliance

Dichiarazione di conflitto di interessi: L'Autore dichiara di non avere conflitto di interessi.

Contributi economici degli autori: L'Autore dichiara di non aver ricevuto sponsorizzazioni economiche per la preparazione dell'articolo.

Indirizzo degli Autori:

Dr.ssa Valentina Paris

Presidente A.N.E.D.

A.N.E.D. Associazione Nazionale Emodializzati - Dialisi e Trapianto - Onlus

Via Hoepli 3

20121 Milano

presidente.aned@aned-onlus.it 